\title{
Automatic Metadata Retrieval from Ancient Manuscripts
}

\author{
Frank Le Bourgeois ${ }^{1}$ and Hala Kaileh ${ }^{2}$ \\ ${ }^{1}$ LIRIS, INSA de Lyon, France \\ Frank.lebourgeois@liris.cnrs.fr \\ http://liris.cnrs.fr/ \\ ${ }^{2}$ ENSSIB-Université Lumière Lyon II, France \\ kaileh@enssib.fr \\ http: //www.enssib.fr
}

\begin{abstract}
The paper presents a document analysis system to retrieve metadata from digitized ancient manuscripts. This platform has been developed to assist researchers, historians and libraries to process a wide variety of manuscripts written in different languages. In order to retrieve different metadata from various digitized documents, we propose a user-training system, which use robust approaches based on a sequential bottom-up process. We develop a low-level segmentation and a basic recognition stage which do not use prior knowledge on documents contents. Our objective was to study the feasibility to process a large variety of manuscripts with the same platform, which can be used by nonspecialists in image analysis.
\end{abstract}

\section{Introduction}

There are many different objectives for the digitization of documents which depend on the final usage of the digital copy [1]. Among the digitized projects, some are concern with the rare medieval manuscripts, which can be accessed only by a reduced number of students and researchers. The digitization of rare collections will improve their accessibility to a wider audience and will protect rare and fragile documents from frequent handling. It also provides to historians, researchers, a new way of indexing, consultation and retrieval of information Medieval manuscripts contain a wide variety of metadata such as text contents, layouts, typography, writing styles, documents structures, author authentication, inks, paper texture, written annotations, signs, drawings, ornaments, decorated frames which help historians to date and authenticate the manuscripts. These metadata are difficult to retrieve manually and a fine indexing would not be possible without better automation by the retroconversion process using image analysis. Metadata for medieval manuscripts are very precisely defined by specialists from the European project MASTER ${ }^{1}$ (Manuscript Access through Standards for Electronic Records).

We present a document analysis system for medieval manuscripts, developed in common with specialists from IRHT, the French Institute on Texts History. This

1 http://xml.coverpages.org/masterGentintr.html

S. Marinai and A. Dengel (Eds.): DAS 2004, LNCS 3163, pp. 75-89, 2004.

(C) Springer-Verlag Berlin Heidelberg 2004 
platform has been developed for researchers, historians and libraries who want to retrieve metadata from these digitized medieval manuscripts. We have chosen the metadata which require an intensive manual work that can be automatically retrieved by an image analysis system. Our objectives consist on studying the feasibility to develop documents images analysis systems suited for medieval manuscripts, which can be used easily by non experts in pattern recognition and image analysis. This tool has been developed in order to help the collaboration with the archivists and historians and make them familiar with pattern recognition systems. It also provides the opportunity to understand user's needs.

In a first section, we present the metadata, defined by specialists, which are possible to retrieve successfully by image analysis systems for both Latin and Arabic manuscripts.

The second section describes the architecture of the document analysis system and the approach used. Because of the complexity of medieval manuscripts and their variability, we have chosen to use simple approaches which do not use prior knowledge on the documents contents. It justifies the choice of a user-training system based on classical bottom-up segmentation.

The last section gives preliminary results which just show the feasibility to retrieve automatically the metadata from medieval manuscripts. We have noticed that the results depend more on the quality of the image rather than the complexity of the contents.

\subsection{Medieval Latin Manuscripts}

The manuscripts indexing needs great expertise which a computer vision system cannot achieved properly. But image analysis can retrieve useful information which can help documents indexing. The French Research Institute on Texts History ${ }^{2}$ is a CNRS laboratory which performs fundamental research on ancient manuscripts anterior to 1500. The IRHT collects and digitizes rare manuscripts with the support of the French ministry of culture. They have also participated to the project MASTER which aims to the specification of metadata for the Latin language manuscript. We have defined different metadata that image analysis systems can retrieve automatically:

- Illuminated objects: decorated objects with gold.

- Main body page: which require considering the main text other than the outside objects like: Annotation, notes, drawings, marks for the manufacturer etc.

- Physical layout: The simplest information needed for finding the book structure. The metadata which are interesting for medieval manuscripts are the text baselines which guide the copyist in writing, the number of the text lines, the number of columns and text justification.

- Miniatures: Pictures or scenes painted on the page.

- Initial: A large initial capital letter usually painted in a contrasting color or illuminated in gold.

2 IRHT: http: //www.irht.cnrs.fr 
- Writing styles: The different style of writing that might exist in the same manuscript.

- Keywords: Important words which define book structures like "incipit". They can be written in different way using different alignments.

- Separating different text colors: Text is handwritten using different colors with alternating red, blue, green and black. The study of color spectrum is also important for research; however, it is achieved by physical and chemical analysis. Nevertheless, a costless characterization of colors can be achieved by image analysis if the camera is well calibrated and color references stored.

- Page references: Page numbers are important to locate and to read automatically in order to detect missing pages, and for indexing each physical image with the correct logical reference of a precise citation.
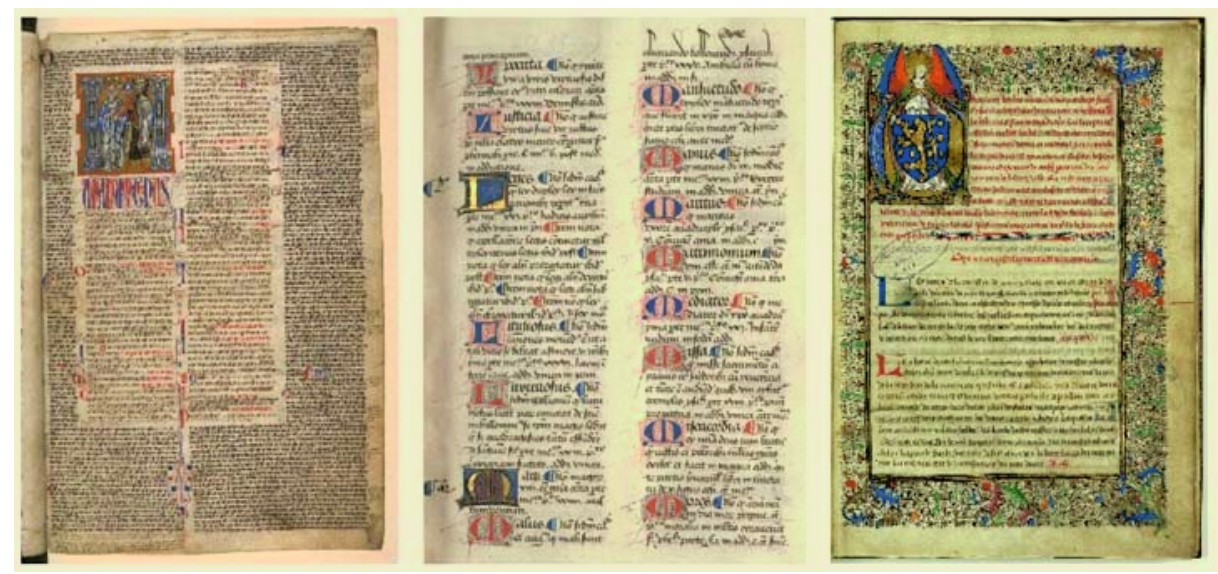

Fig. 1. Medieval manuscripts showing illuminated paintings, initials, decorations, decorated characters, frames, complex layout.

We have discarded from our analysis all metadata which require prior knowledge and specific processing like the physical layout, the reading of page reference and words spotting. On the opposite we keep all the metadata which can be retrieved with a generic approach like the writing styles, colored objects, paintings, illuminated objects, drop capitals and specific character decoration.

\subsection{The Metadata for the Arabic Manuscripts}

The complex nature of the Arabic language is evident in the cursiveness of the text, character overlapping, various character shapes, diacritics and the variety of calligraphic Arabic writing style. Metadata from MASTER project has been extended to Arabic manuscripts [2]. One hundred seventy three elements of metadata were created to cover all the aspects of the Arabic manuscripts. This work was a result of three main studies: 
- A study of the literature that talks about the general characteristics of the Arabic manuscripts from a point of view codicology and palaeography.

- A corpus of 21 digitised manuscripts (3200 images) was thoroughly analysed.

- A questionnaire was distributed among the experts of Arabic manuscripts so as to find out their needs.

The metadata extracted, tried to take into consideration the different aspects of the user's interest, the history of the manuscripts, the codicology, the palaeography, the logical structure etc. We could classify the metadata into three main categories:

a) Bibliographic metadata, which describe the origin of manuscripts (author, title, date, place etc.).

b) metadata that descript content and layout of a manuscript, its logical structure as well as the particular characteristics of the manuscripts such as the existence of illustrations, the figures, the poem lines, the punctuations the stamps, etc.

c) Administrative metadata which record physical information on the manuscript support (microfilm, digitised copy, etc.), condition of digitization, the entry date of the manuscript in the database, the name of the cataloguer etc.

Our concern in this article is to define metadata from the second category which can be retrieved by computer vision systems. They are very similar to metadata defined for Latin manuscripts, but Arabic manuscripts show some specificities:

- Main body page: In the opposite of the Latin manuscripts, most of Arabic manuscripts were written inside a framed page, specially the Koran text.

- The writings on the margin: these writings aims at giving some explanation for the text, comments by the reader or in some cases are used to describe an event or an action like what is known in Arabic by (al-sama'at wa al-qira'at) means the listening and the readings. The writings on the margin are also different in there presentation, some are written vertically or horizontally or in zig-zag etc.

- Ornaments: These important decorations are located in different places of the manuscript: on the margin, inside the text, either to replace the punctuation or around the title of the chapters in some manuscripts.

- The chapter title: They are written either in bold or in different colors. But sometimes the chapter title is written using the same size and color of the text.

- Illustrations: It takes different forms as well as different sizes.

- The page layout: In the opposite of Latin Manuscripts, Arabic manuscripts use generally a single column. But we found two to three columns for Christian-Arabic manuscripts from religious translated text where every column is written in a different language. The writing in a table or in a circle shape is also very common in Arabic manuscripts. The rectangle shape writings are found at the end of the majority of the manuscripts and particular in the colophon where the copyist or the author ends his manuscript by mentioning the place and the date of finishing his work. The three millions Arabic manuscripts that are scattered all around the world holds different other characteristics that it is difficult to present it all in this article. It's worth mentioning here that other manuscripts that still not discover may have other characteristics that tell now not has been covered by the codicological studies. 


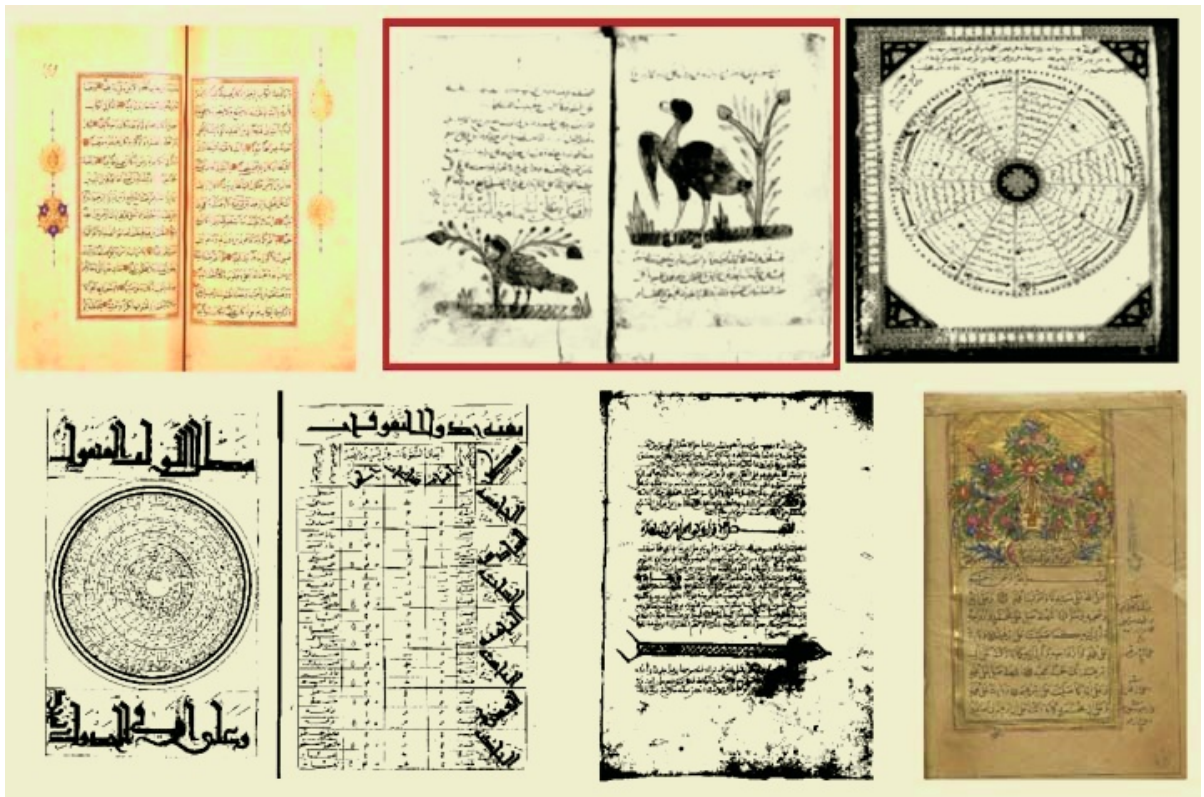

Fig. 2. Samples of Arabic manuscripts and the variety of metadata and images qualities.

\section{The Proposed Document Image Analysis System}

We have developed a generic platform to retrieve information from old manuscripts by supervised training. We found this approach more realistic than a fixed rule-based system which cannot describe the great variety of multilingual manuscripts. Supervised training system allows users to define the metadata and to build their own model for each manuscript. We assume that books have a homogeneous layout and keep a regular presentation of the metadata which can be described by a user built model. We chose a simple sequential architecture which do not require prior knowledge or complex rules. We also reject complex architectures or non deterministic approaches which use feedback loops, heuristics, multi-agents approaches or systems based on interactions. Our objective consists to study the feasibility to process automatically digitized manuscripts by using a generic platform which can be used by non-specialists in image processing and pattern recognition.

\subsection{Image Segmentation}

Image quality of the digitized manuscripts is not regular. To save money and time, numerous projects digitize microfilm copy of the manuscript rather than the originals. The digitization of microfilms is an easy process which is faster and cheaper than using the originals. But in the other hand, it provides bad quality images generally using very few gray levels because the microfilm process clarifies the background and enhances the contrast. The digitization of microfilm is frequently achieved in bi- 
level because information represented by other gray levels is not relevant. Consequently, information loss during the digitization process cannot be restored.

In order to analyze color, grayscale and bi-level images, we propose to convert step-by-step color images into the optimal gray level images then into bi-level images. It allows the analysis of images with different color depths by the same processing line. Between each step, we keep all intermediate images for the image features extraction. Users selects the best algorithm which is immediately stored in the userbuild model associated to the document. We have also developed a specific color segmentation algorithm for digitized documents based on a unsupervised classifier which use local information computed in a sliding window to adapt color variations [3].

As we do not have a precise model of the layout for all medieval manuscripts, we chose a bottom-up segmentation (data-driven), which does not require prior knowledge. We localize first all connected components, and then we classify each component according to the features selected by users. Recognized components are gradually merged into higher interpreted elements, like text, tables, drawings, decoration, frames...
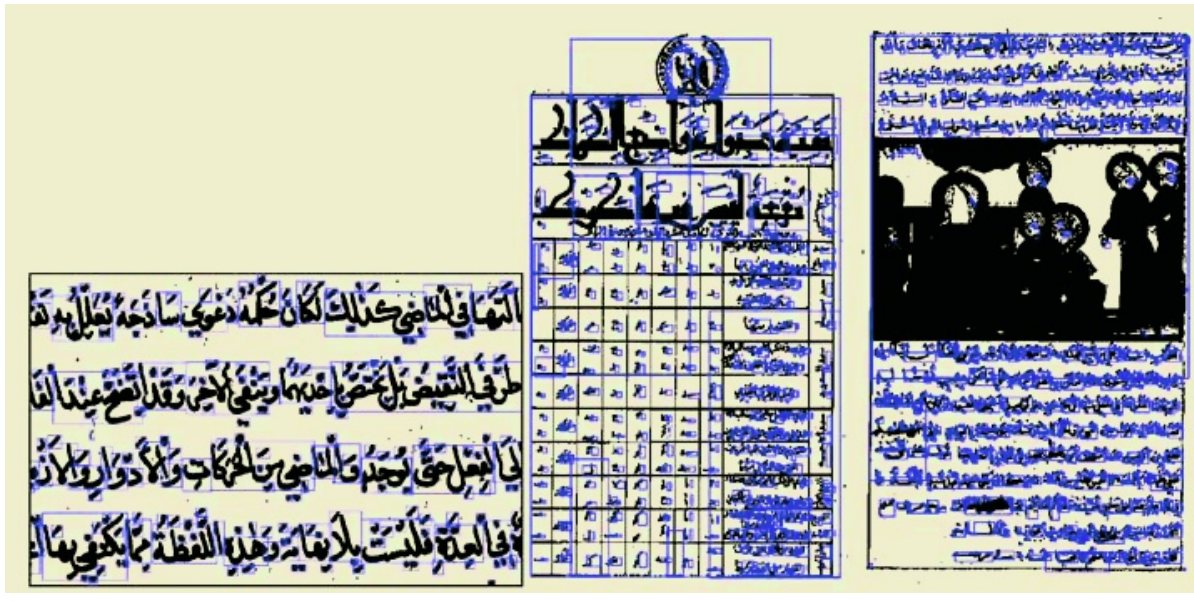

Fig. 3. First step of the bottom-up process : the connected components labeling.

We provide ready-to-use processing tools which remove noise in bi-level images, disconnect touched objects and remove frames. All these tools are based on mathematical morphology [4]. For example, to remove frames and the shadow of the book borders in bi-level images we apply several morphological operations. We measure, by morphological convolution, the Ferret diameter in the vertical direction of each connected component. The resulting images describe each component with a grayscale level corresponding to the height of the minimal convex hull which contains the object (fig 4a). According to a user defined threshold, we build two images, which separate small objects which mostly contain texts (fig 4c) from large objects like frames and pictures (fig. 4d). Then we compute by morphology, the distance transform, to measure the thickness of large objects. Objects having a small thickness and 
the largest in height will be deleted (fig 4e). The final image without frame (fig 4f) is computed by summation of the text image (fig 4c) and picture image (fig 4e). Similar approaches used to disconnect touched components and filter noise of the objects contours are described in [5].

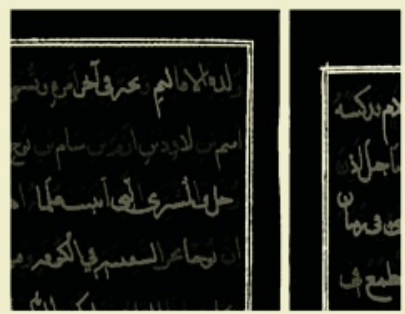

a) Ferret $90^{\circ}$ diameter

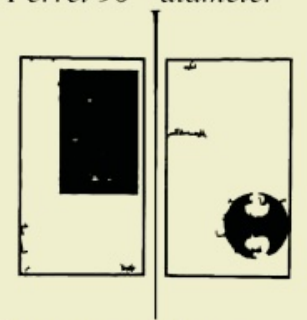

d) Frames and images

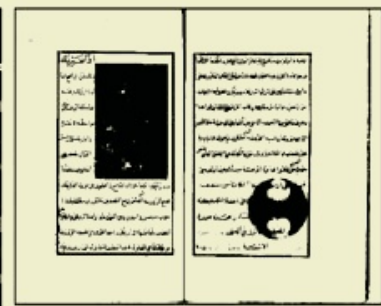

b) original image
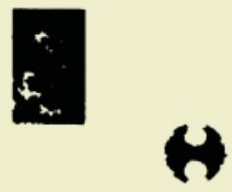

e) Frame suppression

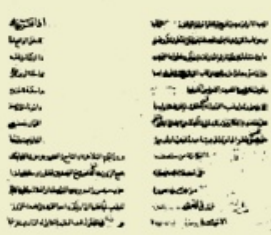

c) Text only

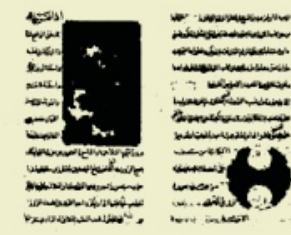

f) Image without frames

Fig. 4. Frames suppression by morphology.

\subsection{Main Body Segmentation}

We have designed other specialized tools to recognize the main body of a page without layout extraction. The location of the main text body is a very important issue because the classification of objects may change according to their position of the main document body. For example, text zone is classified as annotations if it is located outside the main body and regular text if it is inside. The main body may be delimited by a frame, but book borders and some figures which might be considered as frames could introduce some confusion. We choose to detect the main body by locating the text, even though the text is not always justified and filling the entire page. First, we estimate the average size of text symbols by computing the size average of all components. In a second step, we compute a text probability value for each component based on the normalized differences between the size of the component and the average text symbol size (Fig. 5b).

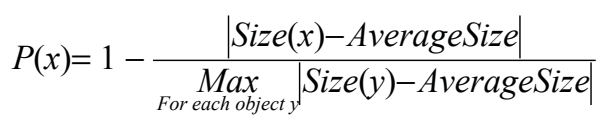

A high value indicates that the component has a high probability to be a text symbol. Then, we sum text probability values horizontally and vertically to build X-Y 

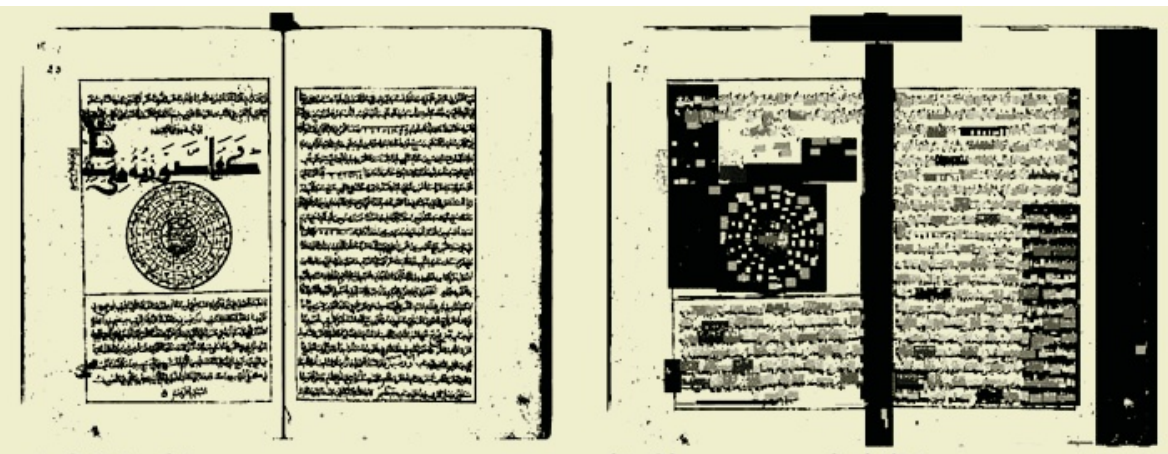

a) Original image

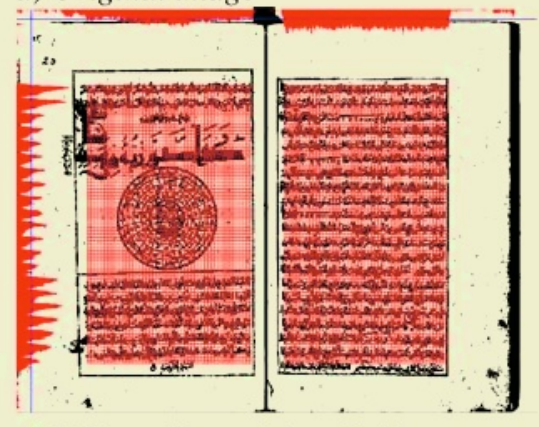

c) $X-Y$ profiles and threshold

b) Class text probabilities

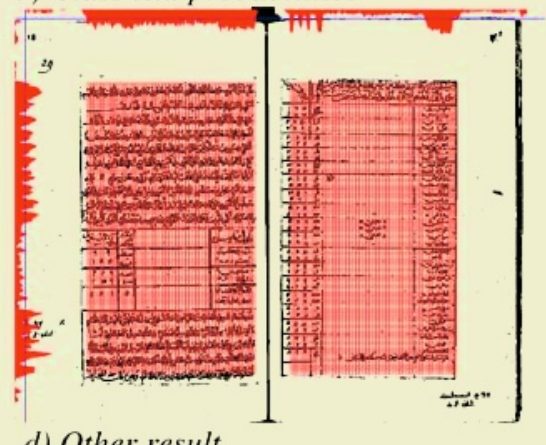

d) Other result

Fig. 5. Main body segmentation using text zones location.

profiles for the entire image. An automatic threshold is computed for each profile. The text bodies, for two pages of one document, are found by taking the maximum limits of the profile coordinate having values lower than the computed threshold (Fig. 5c). This approach works most of the time, even on Latin manuscripts, but the text bodies can be oversized in the case of large annotations areas in the margin.

\subsection{Feature Analysis}

After document layout analysis in terms of columns, lines and words, we compute for each component a feature vector using measures selected by the users and which depends on the metadata to be retrieved and the complexity of the image. We have chosen 3 families of features:

Color features (RGB, YUV, HSL color systems)

Shape features (object density, compactness, convexity, anisotropy, orientation, contour, moments, curvature distribution of the contour, X-Y profiles) (Fig. 6)

Geometry (object size, maximum diameter in 8 directions, thickness, length...) 


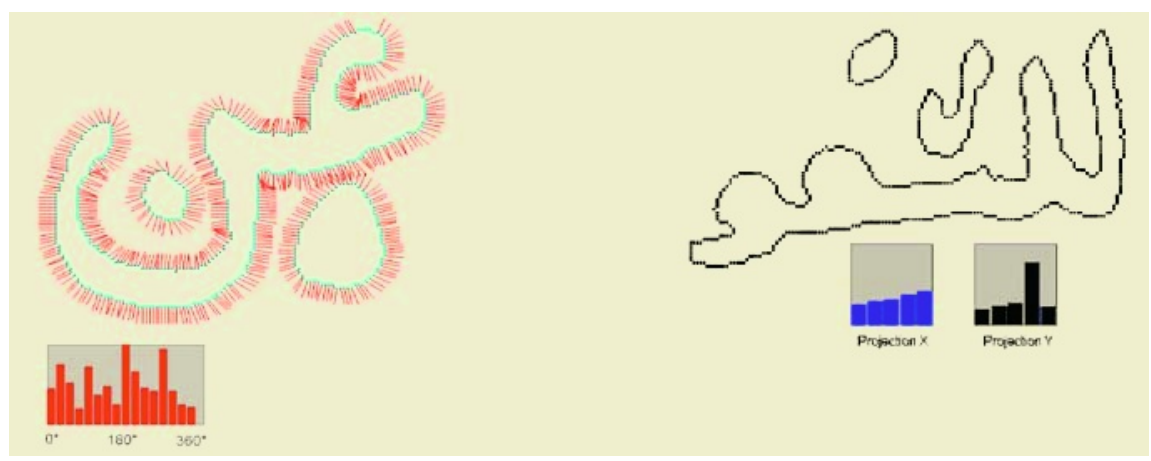

Fig. 6. a) Curvatures distribution along the contour b) X-Y projections.

The recognition of some metadata is not only based on the objects shape, size and color, we have to take also into account spatial relations between objects. For example, alignments and regularity of words positions are important to recognize text from tables. Some chapter titles are recognizable only by the indentation when they use the same style and the same size of the regular text. Alignments and distances between objects are important features to consider for many metadata. We have measured two horizontal alignments $\boldsymbol{a} \boldsymbol{h}$ with the left and right neighbours, two vertical alignments $a v$ with the top and bottom neighbours and four distances $\boldsymbol{d}$ with the four nearest neighbours (Fig. 7). Contrary to the Latin manuscripts, we measure the vertical alignments $\boldsymbol{a} \boldsymbol{v}$ for the Arabic manuscripts, on the right side, in order to consider the reverse direction of reading.

To take into account the recognition of neighboring objects, we choose to augment the feature vector of each component with the features of neighboring objects in the four main directions. The dimension of the feature space is too high to build a classifier with a limited set of training vectors. We reduce the dimension of the feature space with an automatic feature combination algorithm using the Principal Components Analysis (PCA). This stage also avoids the user to arbitrary combine or to select the features which maximize the clusters separation.

a)

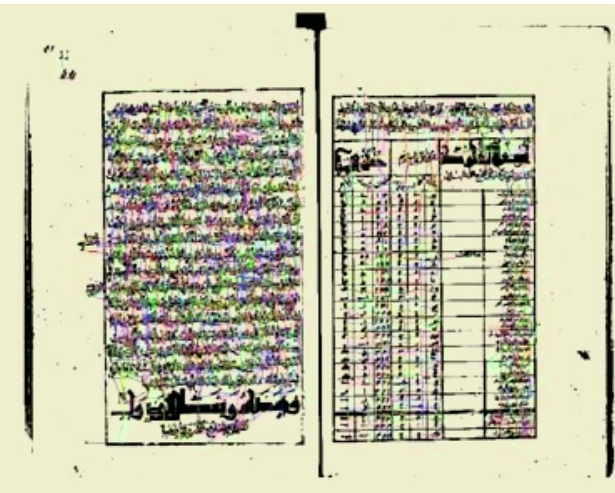

b)

Fig. 7. a) Spatial relations between objects; b) Spatial features $\boldsymbol{d}, \boldsymbol{a h}, \boldsymbol{a} \boldsymbol{v}$. 


\subsection{Training and Recognition}

Training is an important step which is achieved easily by using a friendly interface. The user defines the number of classes and gives a class name for each metadata. The interface, figure 15, allows selecting components and their classes of metadata. All corrections or new entries are saved in the model which displays the number of training vectors for each class of metadata. The training step is accomplished by using several representative pages from the same manuscript. The model associated with each manuscript store the training vectors, the number of classes of metadata and their names, the color conversion process, the thresholding method, the postprocessing operations (frame removal, disconnection of touched objects, noise removal) and the features selected by users. We chose a simple K-NN classifier for its training speed and for its simplicity. A condensing algorithm is used to reduce the number of vectors and improve the recognition speed without decreasing the performance. We are aiming to use a more appropriated classifier like the SVM, suitable to the reduced number of training vectors, but this approach requires a longer training step. Recognition is achieved for all connected components segmented within the image. We consider each object differently, according to its location inside or outside the main page body, in the resulting file.

\section{Experiments and Results}

The platform has been experimented on 6 Arabic manuscripts and 2 Latin manuscripts which make up 1361 single pages of manuscripts. The number of classes of metadata differs from a manuscript to another. The recognition results are difficult to evaluate precisely because of the lack of ground-truth in this field. A preliminary coarse evaluation of the results is enough to determine the feasibility of the proposed document analysis system. We notice that the results depend more on the quality of image rather than the origin of the manuscripts and its language. We present the results both for Latin and Arabic manuscripts classified by image quality. For color images directly scanned from the originals, using high resolution, the results are very satisfactory and we had obtained successful recognition of a large variety of metadata like titles, ornaments, drawings, text delimiters, colored objects, punctuation and regular text. The following figures show results for both Arabic and Latin colored manuscripts.

For the given sample of Arabic colored manuscript (fig. 8a), we have defined 4 logical classes: regular text (fig. 8d), notes (fig. 8c), illuminated ornaments and frames (fig. 8f) and punctuation (illuminated circles(fig. 8e)). The difference between principal text and notes is based on the positions of objects from the respect to the main document body.

The second example shows different metadata from medieval French manuscripts. We have selected very few features like the RGB color system as color features and the thickness and average curvatures of components as shape features. 


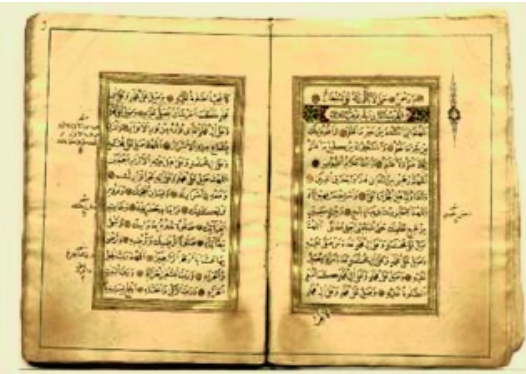

a) Original Image

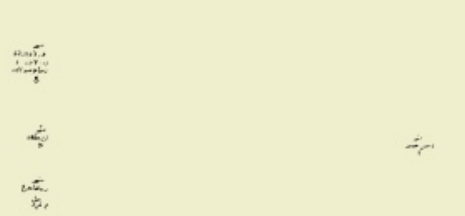

c) Notes

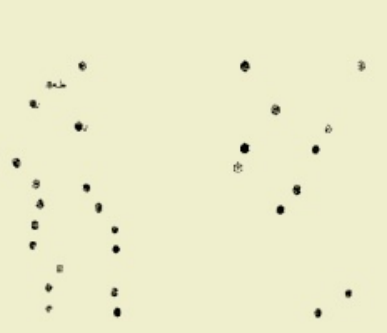

e) Illuminated punctuation

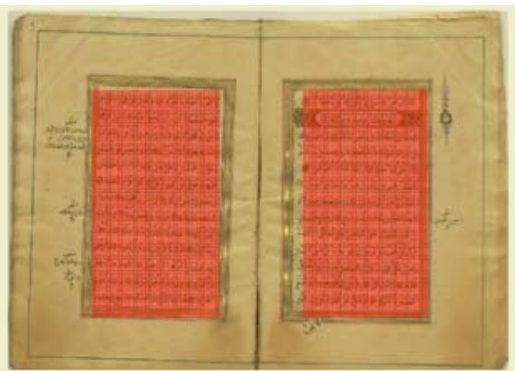

b) Main body localization

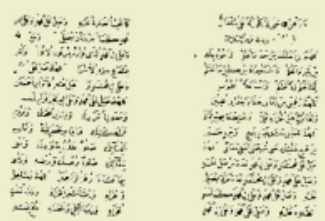

d) Principal Text

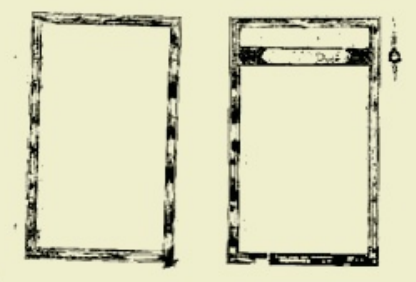

f) Illuminated frames and drawings

Fig. 8. Results of metadata extraction into manuscripts from Palestine MS6191 (BM Lyon).

These simple features are sufficient to perform recognition with a very high accuracy: the original text from the main copyist, the additional writings by the second copyist and the colored drop capitals (fig. 9). From these documents, we also recognize easily the paintings, the illuminated ornaments, the special words written with thick lines and text baselines which help the copyist to align the text. Last example shows that even for complex and rich documents, the bottom-up approach make possible to segment several hundred objects in the same page and classify them correctly by using simple features like the color (illuminated and red characters or miniatures), the thinness and average curvatures of objects (difference of writing style). But the circular stamp from the library located in the center of the page are not correctly classified because of the thickness of the components. 


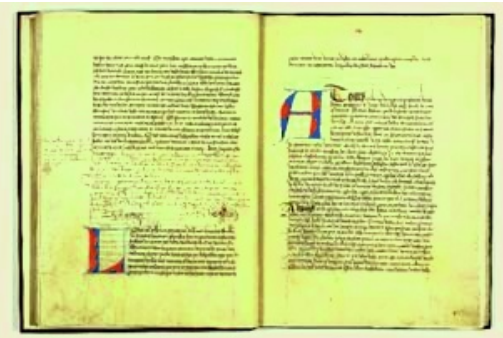

a) Original Latin Manuscripts

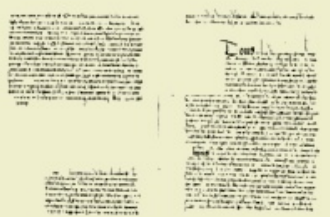

b) Original Text from the main copyist

\section{H}

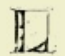

d) text from the second copyist

e) Colored drop capitals

Fig. 9. Results of metadata extraction into manuscripts from Auxerre AA01 source IRHT.

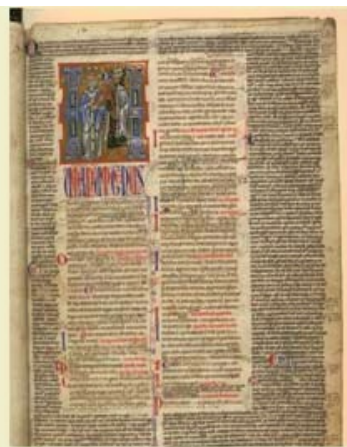

Original image

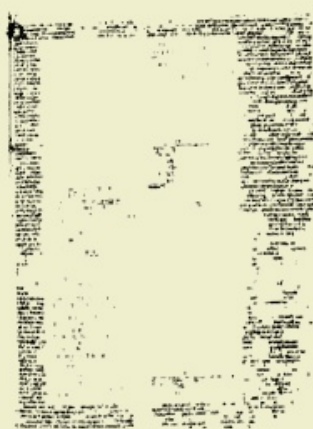

First writing (bold)

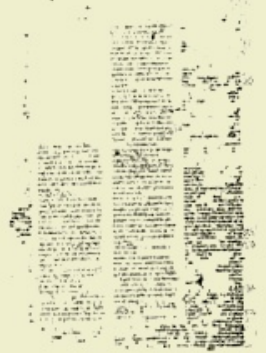

Second writing (thin)

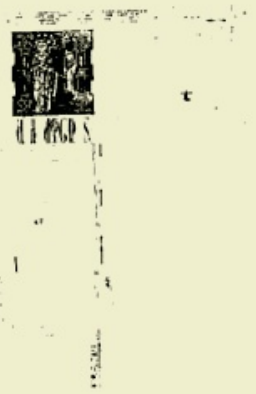

Blue objects

Red objects

Fig. 10. Results of metadata extraction into manuscripts from Amiens MS0354 source IRHT. 


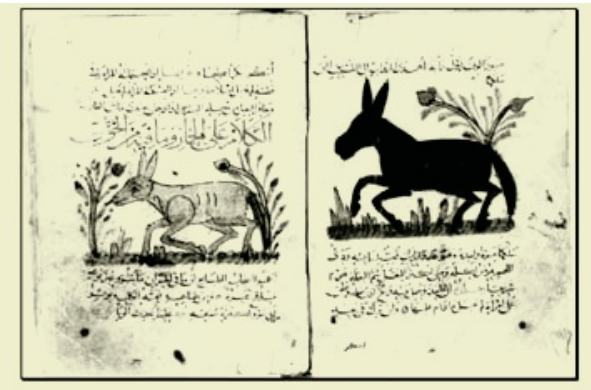

Original grayscale image

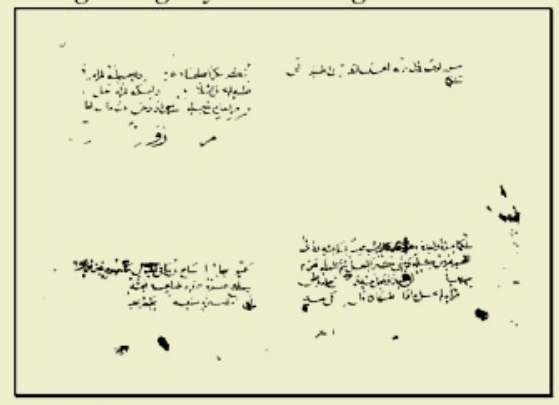

Black text
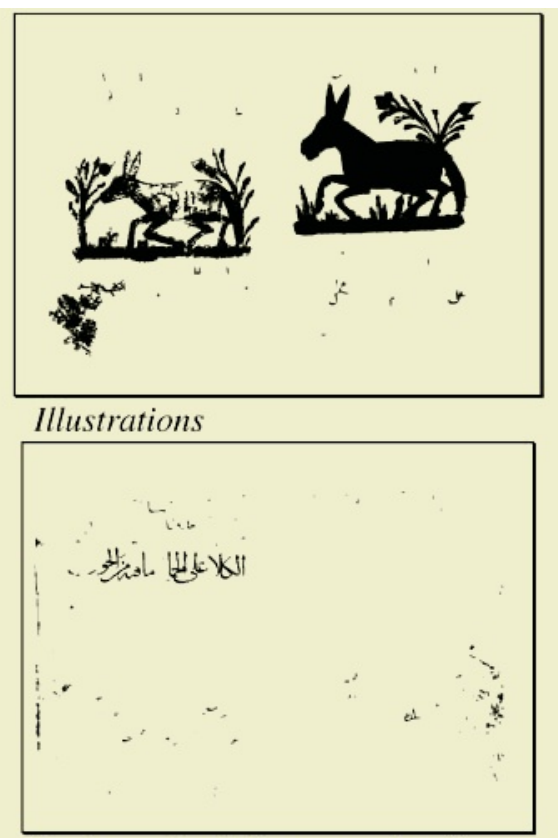

Red text (in lighter gray level)

Fig. 11. Results of metadata extraction into manuscripts R12051 from BNF.
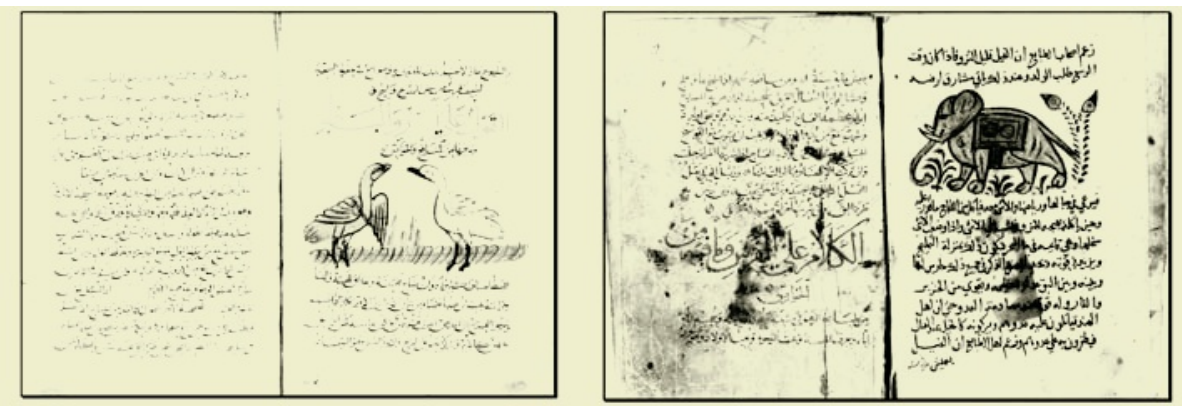

Fig. 12. Errors from manuscripts R12051 due to the over-exposure or the stains.

For grayscale or binary images scanned from microfilms, the results depend on the quality of the support (stains, holes, color of the ink...), the using or not of the JPEG compression, the resolution used and the regularity of the image contrast and brightness.

The main difficulty with the microfilm digitization is the lack of the regularity of exposure and the enhancement of the contrast, which reduce the number of grayscale levels, after brightness normalization. For the manuscripts R12051 (fig. 11) digitised from microfilms, the quality of images is not sufficient to retrieve correctly illustrations because of stains and to differentiate black and red texts because of the brightness variation for the same manuscripts (fig. 12). 

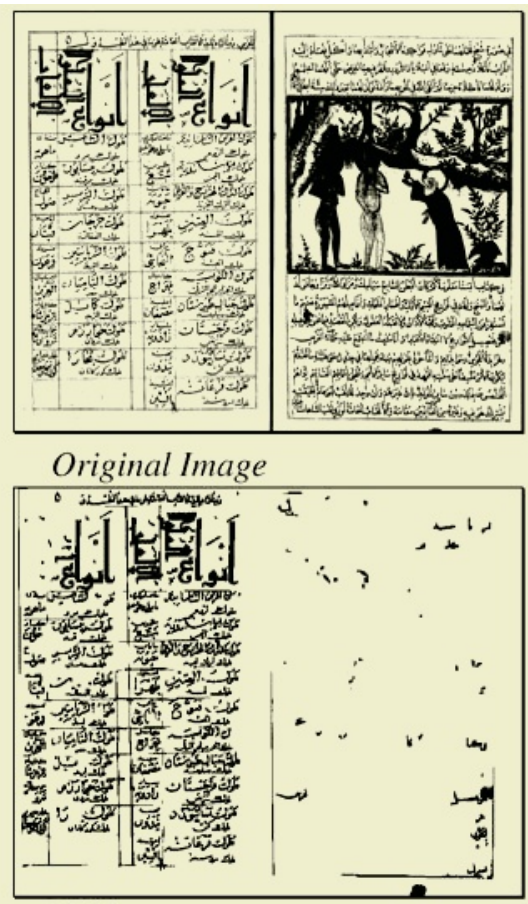

Tables
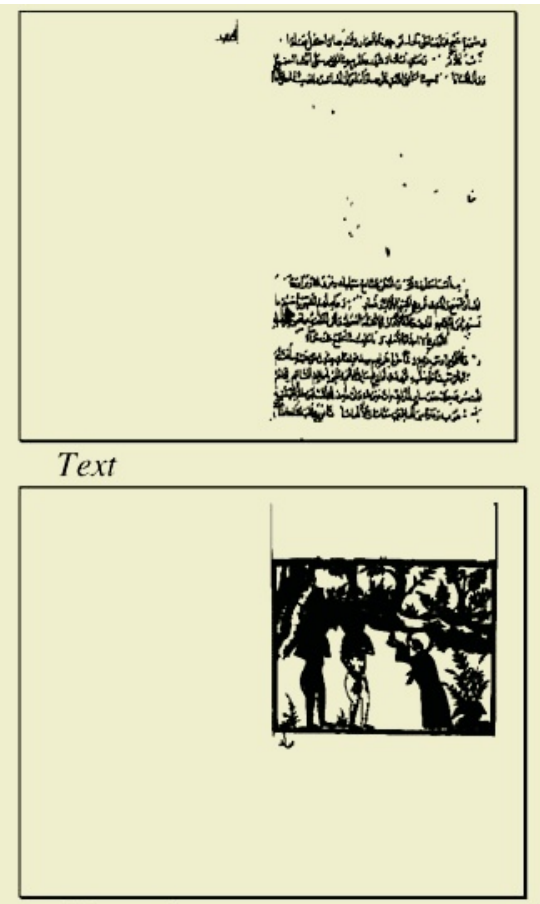

Illustrations

Fig. 13. Results of metadata extraction into manuscripts R28062 from BNF.

For the more complex manuscript we have to analyze (R28062 from BNF), the quality of the binary image is sufficient to retrieve simple metadata like illustrations, tables, text and titles. But our platform cannot process combined metadata. In the previous example, it is impossible to make a difference between titles inside tables and titles outside tables because they have the same shape and almost the same spatial relations. In order to achieve this separation, we have to recognize first the object 'table' and then the object 'title in the table'. A cascade architecture is required to retrieve combined metadata.

Figure 14 shows that complex metadata like circular tables cannot be recognized properly. After the merging step which group progressively connected components from the same label, the system finds that the circular table is classified both as illustration and table. Moreover some random alignments of the diacritics of the titles and the numerals include in the text are systematically classified as tables. It shows the limit of our simple architecture which cannot retrieve complex metadata without specialized algorithms and specific heuristics.

\section{Conclusion and Perspective}

We have developed a DIA system based on a sequential bottom-up process to retrieve simple metadata from ancient manuscripts. The approach chosen limit the perform- 


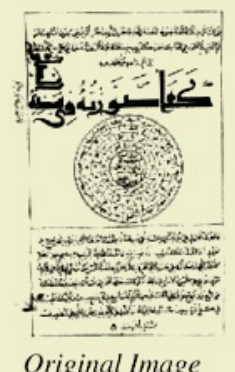

Original Image
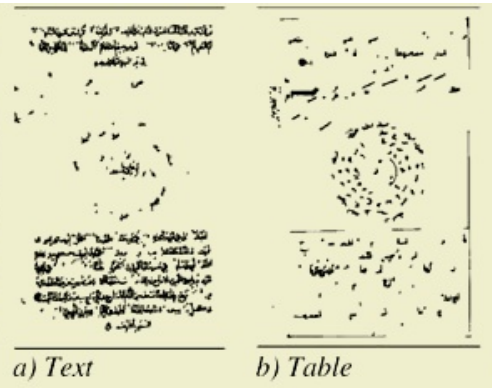

b) Table

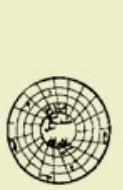

c) Illustration

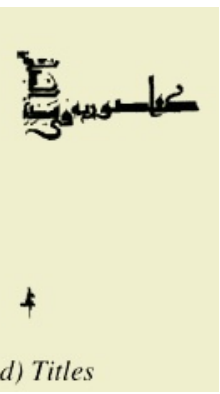

d) Titles

Fig. 14. Results of metadata extraction into manuscripts R28062 from BNF.

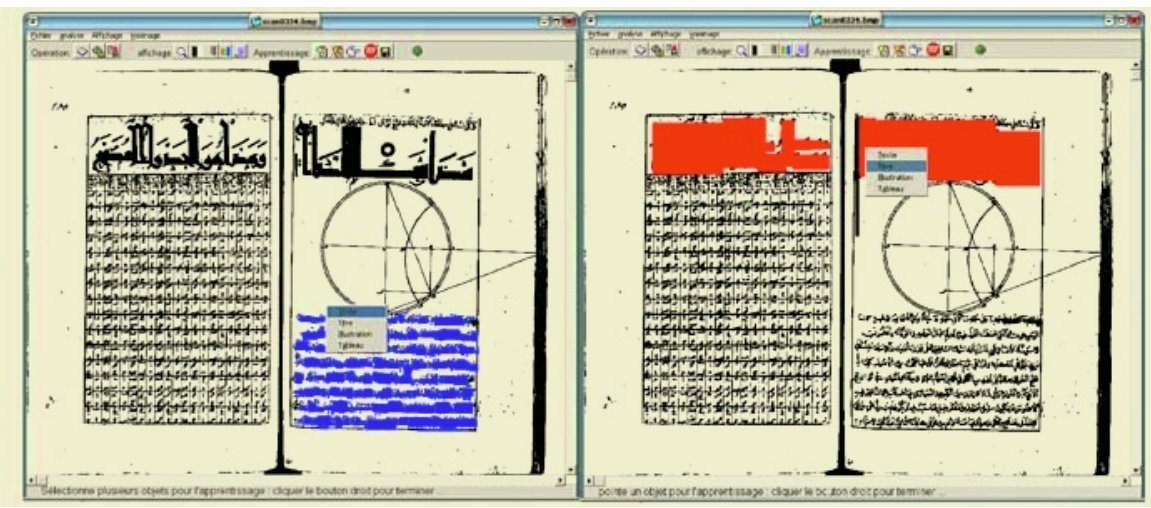

Fig. 15. Interface during the learning step for the training of class "text" and "title".

ance of such tools for complex metadata and bad quality images. Nevertheless, the results are promising for images which use sufficient resolution and color depth. This approach seems generic for simple metadata but many metadata are still not retrieved with our platform like interleaved metadata. So the next step consists on developing a cascade architecture to recognize interleaved metadata. We have also noticed two major problems during the usage of our package: the problem for users to select incomprehensible and complex parameters during the model creation and the time consuming training step. The productivity of such tools must be evaluated by users in the long-term and ergonomic must be improved for better usage by neophytes.

\section{References}

1. LeBourgeois, F., and al., Document Images Analysis Solutions for Digital Libraries, Proc. Of the DIAL 2004, 23-24 jan. 2004, Palo Alto, California, pp 2-24.

2. Kaileh, Hala., L'accès à distance aux manucrits arabes numérisés en mode image. $\mathrm{PhD}$, university Lyon II, France, January $28^{\text {th }}$ 2004, 445p.

3. Leydier Y., and al., Serialized k-means for adaptative color image segmentation, submit to DAS 2004

4. Serra, J., Image analysis and mathematical morphology, Acedemic press, London, 1982.

5. Bres, S., Jolion, J-M., Le Bourgeois, F., Traitement et analyse des images numériques, Hermes, 2003, $412 \mathrm{p}$. 